\title{
可见光介导无金属的苯乙酸衍生物脱羧氞代
}

\author{
何宇航杨慧高冬旭马嘉慧郡亚敏 \\ 安光辉* 李光明* \\ (黑龙江大学化学化工与材料学院 功能无机材料化学重点实验室＼cjkstart哈尔滨 150080)
}

\author{
摘要 报道了无金属可见光诱导的苯乙酸衍生物脱羧. 在无添加剂条件下，使用廉价的氞源成功地实现了酸的直接脱 \\ 羧单気化. 此外, 在空气存在时, 类似的催化体系能够通过脱羧氧化生成酮. \\ 关键词 光化学; 无金属; 脱羧; 氛代
}

\section{Visible Light-Mediated Metal-Free Decarboxylative Deuteration of Carboxylic Acid}

\author{
He, Yuhang Yang, Hui Gao, Dongxu Ma, Jiahui Shao, Yamin \\ An, Guanghui* Li, Guangming* \\ (Key Laboratory of Functional Inorganic Material Chemistry, School of Chemistry and \\ Materials Science, Heilongjiang University, Harbin 150080)
}

\begin{abstract}
A metal-free visible light-induced decarboxylation of phenylacetic acid derivatives has been successfully developed. Without the additives, direct decarboxylative monodeuteration of acids was successfully achieved using cheap deuterium sources. Besides, in presence of air, similar catalytic systems provided ketone products.

Keywords photochemistry; metal-free; decarboxylation; deuteration
\end{abstract}

\section{Introduction}

Introduction of deuterium labels is important method to detect and quantify drugs and drug metabolites both in vivo and in vitro. ${ }^{[1]}$ With the approval of the first deuterated drug (Austedo) in 2017 by the Food and Drug Administration (FDA), deuterated compounds were found to potentially affect the metabolic sites and improve the pharmacokinetic properties. Mono-deuterated methyl compound has been regarded as an important tool not only in drug discovery and development, but also in mechanism investigations. ${ }^{[2]}$ However, in contrast to $\mathrm{CD}_{3}$ group, the current methods for preparing mono-deuterated methyl groups are limited. To date, reported labeling approaches mainly include transition metal catalyzed H/D exchange,,$^{[3]}$ and reduction and deuteration of olefins or carbonyl groups. ${ }^{[4]}$ They normally require harsh conditions with transition metal catalysts or stoichiometric reductant, generating corresponding wastes. $^{[5]}$ Thus, the development of environmental-benign metal-free protocols to install mono-deuterated methyl would be highly demanding.

Carboxylic acids are common commercially available feedstocks. ${ }^{[6]}$ In view of the diverse skeletons of carboxylic acids, decarboxylative deuteration represents an elegant access to deuterated molecules from commercially available starting materials, compared with well-developed radical dehalogenative deuteration of organic halides. ${ }^{[7]}$ Oshima et $a l .{ }^{[8]}$ reported Pd-catalyzed decarboxylative deuteration of aliphatic carboxylic acids, providing fully deuterated hydrocarbons. Gooßen et al ${ }^{[9]}$ disclosed $\mathrm{Cu}$ or Ag-catalyzed decarboxylative deuteration of (hetero)aromatic carboxylic acids at elevated temperatures. Besides these thermal reactions, the recent emerging photoredox catalysis ${ }^{[10]}$ was also developed for novel decarboxylative deuteration of acids. Glorius and colleagues ${ }^{[11]}$ developed the deuteration of oxime carboxylates catalyzed by $\left[\operatorname{Ir}\left(\mathrm{dF}\left(\mathrm{CF}_{3}\right) \mathrm{ppy}\right)_{2}(\mathrm{dtbb}-\right.$ py) $]\left(\mathrm{PF}_{6}\right)$. Xie and others ${ }^{[12]}$ employed thiols as in situ generated deuterated reagents for decarboxylative monodeuteration. Wang et al. ${ }^{[13]}$ disclosed ceramic boron carbonitride $(\mathrm{BCN})$ catalyzed decarboxylation of carboxylic

* Corresponding authors. E-mail: chemagh@163.com; gmli@hlju.edu.cn

Received November 6, 2021; revised December 11, 2021; published online December 15, 2021.

Project supported by the National Natural Science Foundation of China (No. 21502046).

国家自然科学基金(No. 21502046)资助项目. 
(a) Previous works: decarboxylative deuteration of carboxylic acids

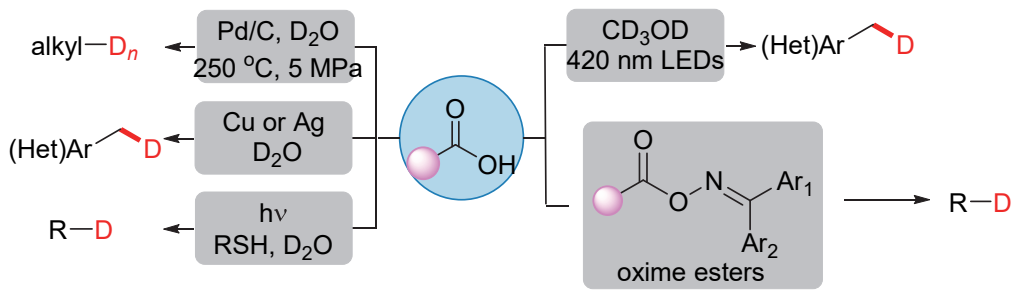

(b) This work: decarboxylative deuteration and oxygenation of carboxylic acids

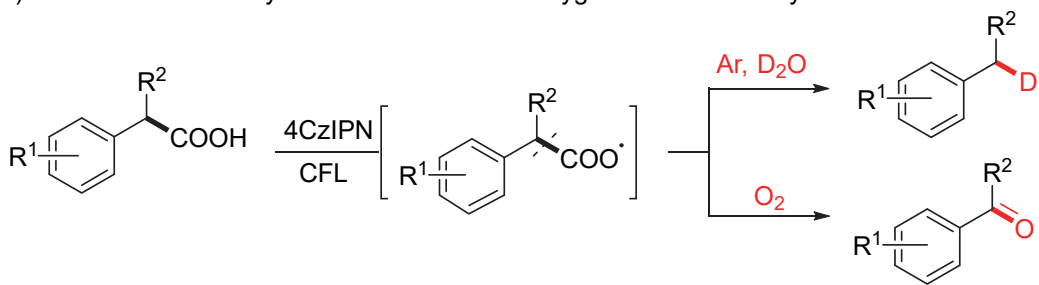

Scheme 1 Challenges and current work of decarboxylative deuteration

acids using $\mathrm{CD}_{3} \mathrm{OD}$ as deuterated reagent (Scheme 1a).

Despite these great efforts, an additive free selective decarboxylative monodeuteration using cheap deuterium sources remains unexplored. Herein, we disclosed a metal-free visible light-induced decarboxylation of phenylacetic acid derivatives (Scheme $1 \mathrm{~b}$ ). Without the additives, direct decarboxylative monodeuteration of acids was successfully achieved using cheap deuterium sources. Besides, in presence of air, similar catalytic systems provided ketone products. Notably, these protocols were viable to many medicines.

\section{Results and discussion}

We started our study by reacting naproxen 1a, a nonsteroidal antiinflammatory drug, with $\mathrm{D}_{2} \mathrm{O}$ in dimethyl sulfoxide (DMSO) employing various photoredox catalysts in the presence of $\mathrm{Cs}_{2} \mathrm{CO}_{3}$ as a base under argon (Table 1). 2,4,5,6-Tetra(9H-carbazol-9-yl)isophthalonitrile (4CzIPN), an important organic light-emitting diodes material with thermally activated delayed fluorescence, ${ }^{[14]}$ proved the most efficient photocatalyst in the decaboxylative monodeuteration, providing desired product $\mathbf{2 a}$ in $63 \%$ (Entry 1 vs. Entries 2 3; for details see Supporting Information, Table S1, Entries 1 9). Furthermore, the yield of 2a significantly decreased in absence of 4CzIPN, illustrating the crucial role of 4CzIPN (Entry 4). During further survey of different bases, $\mathrm{Cs}_{2} \mathrm{CO}_{3}$ afforded the best reactivity (Table 1, Entry 1 vs. Entries $5 \sim 8$ ). Increasing the amount of base did not promoted the reaction efficiency (Table 1, Entry 9). Investigation of the solvent proved that DMSO was optimal for the reaction (Table 1, Entry 1 vs. Entries $10 \sim 14)$. Notably, ketone 3a together with small amount of 2a was obtained under air (Table 1, Entry 15).

Subsequently, the decarboxylative oxygenation reaction of phenylacetic acid derivatives has been optimized under air (Table 1, Entry 16 vs. Entries 17 20; for details see Supporting Information, Tables S4, S5 and S6). Gratifyingly, when $1.0 \mathrm{~mL}$ of DMSO was used as the solvent and
$\mathrm{Cs}_{2} \mathrm{CO}_{3}$ was used as the base, it proved to be the best condition to provide the ketone product 3a (Table 1, Entry 16). Notably, the reaction afforded slightly higher yield under oxygen (Table 1, Entry 21).

With the optimized conditions in hand, the scope of the carboxylic acids was investigated (Table 2). Similar to 1a, several secondary acids were subjected to the standard conditions. These biaryl carboxylic acids underwent decaboxylative monodeuteration smoothly to give the desired products $\mathbf{2 b} \sim \mathbf{2 d}$. This protocol would be accessible to primary carboxylic acids with para-amide or phenyl groups as well, albeit in low yields $(\mathbf{2 e}, \mathbf{2 f})$. Furthermore, the synthetic robustness of the reaction was illustrated by the decarboxylative deuteration of complex carboxylic acids such as ketoprofen, carprofen, isoxepac and indomethacin $\mathbf{2} \mathbf{g} \sim$ 2j). Notably, substrates with electron-rich $N$-heteroarenes (indole) exhibited lower reactivity than that with electron-deficient benzophenone moiety.

Next, the scope of the decarboxylative oxygenation reaction was examined (Table 3 ). Secondary biaryl carboxylic acids with ether moiety exhibited higher reactivity over others (3a, 3d vs. 3b, 3c). Primary carboxylic acids with para-amide or heterocyclic substituents were well tolerated, giving products $\mathbf{3 e}$ and $\mathbf{3 f}$ in $19 \%$ and $44 \%$ yields. It is worth noting that the ether structure-containing substrates showed higher reactivity than the amide-substituted substrates. This methodology can be utilized for the transformation of various pharmaceuticals with functionalized phenylacetic acid scaffolds. Ketoprofen, $(S)$ - $(+)$-ibupro- fen, flubiprofen and isoxepac were all transformed into the corresponding ketones and aldehydes $(\mathbf{3 g} \sim \mathbf{3} \mathbf{j})$. Unexpectedly, the product $\mathbf{3 k}$ was obtained from indomethacin.

To gain insights into the mechanism, a series of control experiments were carried out. Addition of 2,2,6,6-tetramethyl-1-piperidinyloxy (TEMPO) successfully inhibited production of both deuterated $\mathbf{2 a}$ and ketone $\mathbf{3 a}$. Furthermore, TEMPO adduct $\mathbf{4}$ was obtained in both conditions, confirming the existence of free radical $\mathbf{B}$ (Scheme 2). As 
Table 1 Optimization of the decarboxylative deuteration and oxygenation reaction conditions ${ }^{a}$

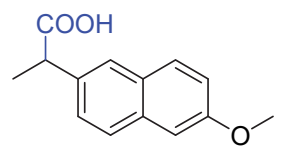

$1 \mathrm{a}$

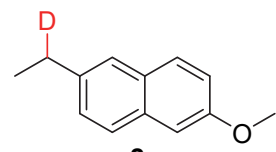

$2 a$

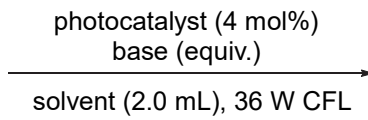

$\mathrm{D}_{2} \mathrm{O}, 24$ h, r.t., $\mathrm{Ar}$

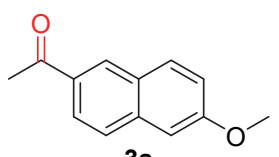

$3 a$

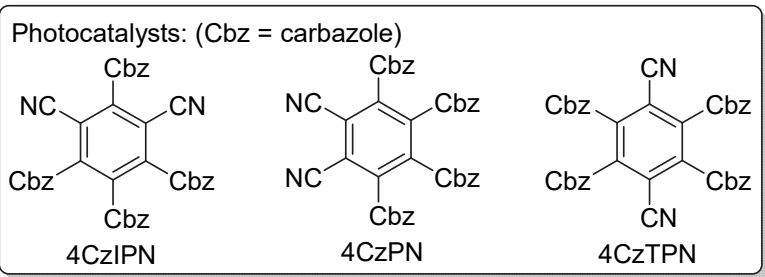

\begin{tabular}{|c|c|c|c|}
\hline Entry & Base & Solvent & Yield $^{b} / \%$ of $\mathbf{2 a} / \mathbf{3 a}$ \\
\hline 1 & $\mathrm{Cs}_{2} \mathrm{CO}_{3}$ & DMSO & $63 /-$ \\
\hline $2^{c}$ & $\mathrm{Cs}_{2} \mathrm{CO}_{3}$ & DMSO & $12 /-$ \\
\hline $3^{d}$ & $\mathrm{Cs}_{2} \mathrm{CO}_{3}$ & DMSO & $5 /-$ \\
\hline $4^{e}$ & $\mathrm{Cs}_{2} \mathrm{CO}_{3}$ & DMSO & $8 /-$ \\
\hline 5 & $\mathrm{~K}_{2} \mathrm{CO}_{3}$ & DMSO & $6 /-$ \\
\hline 6 & $\mathrm{CsOAc}$ & DMSO & $28 /-$ \\
\hline 7 & $\mathrm{CsF}$ & DMSO & $55 /-$ \\
\hline 8 & $\mathrm{CsHCO}_{3}$ & DMSO & $22 /-$ \\
\hline $9 f$ & $\mathrm{Cs}_{2} \mathrm{CO}_{3}$ & DMSO & $60 /-$ \\
\hline 10 & $\mathrm{Cs}_{2} \mathrm{CO}_{3}$ & DMF & $43 /-$ \\
\hline 11 & $\mathrm{Cs}_{2} \mathrm{CO}_{3}$ & $\mathrm{MeCN}$ & $34 /-$ \\
\hline 12 & $\mathrm{Cs}_{2} \mathrm{CO}_{3}$ & DCE & $27 /-$ \\
\hline 13 & $\mathrm{Cs}_{2} \mathrm{CO}_{3}$ & MeCOOEt & $9 /-$ \\
\hline 14 & $\mathrm{Cs}_{2} \mathrm{CO}_{3}$ & $\mathrm{CH}_{2} \mathrm{Cl}_{2}$ & $7 /-$ \\
\hline $15^{g}$ & $\mathrm{Cs}_{2} \mathrm{CO}_{3}$ & DMSO & $5 / 20$ \\
\hline $16^{h}$ & $\mathrm{Cs}_{2} \mathrm{CO}_{3}$ & DMSO & -150 \\
\hline $17^{h}$ & $\mathrm{CsOAc}$ & DMSO & $-/ 15$ \\
\hline $18^{h}$ & $\mathrm{CsF}$ & DMSO & $-/ 19$ \\
\hline $19^{h}$ & $\mathrm{Cs}_{2} \mathrm{CO}_{3}$ & $\mathrm{MeCN}$ & $-/ 16$ \\
\hline $20^{h}$ & $\mathrm{Cs}_{2} \mathrm{CO}_{3}$ & DMF & $-/ 18$ \\
\hline $21^{i}$ & $\mathrm{Cs}_{2} \mathrm{CO}_{3}$ & DMSO & -154 \\
\hline
\end{tabular}

${ }^{a}$ Reaction conditions: 1a $\left(0.1 \mathrm{mmol}, 1.0\right.$ equiv.), $\mathrm{D}_{2} \mathrm{O}$ (3.0 mmol, 30 equiv.), $4 \mathrm{CzIPN}(4 \mathrm{~mol} \%)$, and $\mathrm{Cs}_{2} \mathrm{CO}_{3}$ (2.0 equiv.) in DMSO $(2.0 \mathrm{~mL})$ under $\mathrm{Ar}$ reaction $24 \mathrm{~h} .{ }^{b}$ Isolated products. ${ }^{c}$ Using $4 \mathrm{CzPN} .{ }^{d}$ Using $4 \mathrm{CzTPN} .{ }^{e}$ Without adding photocatalyst. ${ }^{f} 6.0$ equiv of base was added. ${ }^{g}$ Under air. ${ }^{h}$ Without adding $\mathrm{D}_{2} \mathrm{O}$, under air, in DMSO $(1.0 \mathrm{~mL}) .{ }^{i}$ Without adding $\mathrm{D}_{2} \mathrm{O}$, under oxygen, in DMSO $(1.0 \mathrm{~mL})$. DMSO $=$ dimethyl sulfoxide, $\mathrm{DMF}=N, N$-dimethylformamide, $\mathrm{DCE}=1,2$-dichloroethane.

the decarboxylative oxygenation didn't occur under argon (Table 1, Entry 1) and oxygen slightly promoted the reaction outcome (Table 1, Entry 21), we proposed that the oxygen would be crucial as oxidant for decarboxylative oxygenation. According to the experimental results and previous reports, ${ }^{[14 e, 15]}$ a possible mechanism is proposed in Scheme 3. Upon irradiation, 4 CzIPN can readily oxidize 1a by excited state $4 \mathrm{CzIPN} *$, generating alkyl radical $\mathbf{B}$ via elimination of carbon dioxide. In absence of oxygen, sub-
Table 2 Deuteration of phenylacetic acid derivatives<smiles>[R]c1ccc(C([R])C(=O)O)cc1</smiles>

(a) Secondary acid

2a, $63 \%$<smiles>[2H]C1c2ccccc2Oc2ccccc21</smiles>

2d, $76 \%$

(c) Complex acid<smiles>[2H]C(C)c1cccc(C(=O)c2ccccc2)c1</smiles>

2g, $(83 \%)^{b}$

(From ketoprofen)

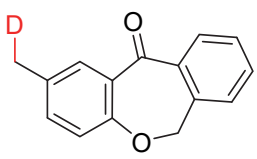

2i, $50 \%$

(From isoxepac)<smiles>[2H]C(c1ccccc1)c1ccccc1</smiles>

2b, $66 \%$

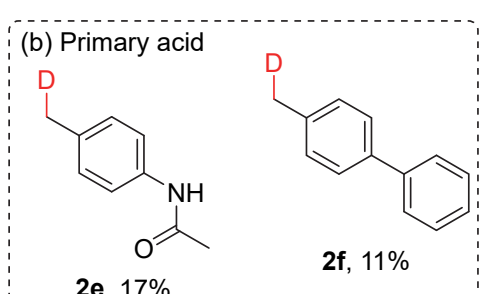

2e, $17 \%$

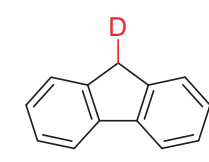

2c, $77 \%$

D

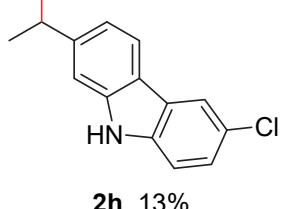

2h, $13 \%$

(From carprofen)

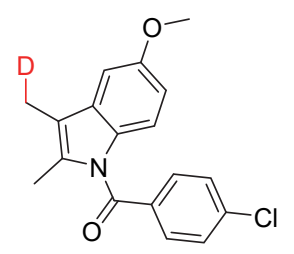

2j, $11 \%$

(From indometacin) a Reaction conditions: 1 (0.1 mmol), $\mathrm{D}_{2} \mathrm{O}(3.0 \mathrm{mmol}), 4 \mathrm{CzIPN}(4 \mathrm{~mol} \%)$, $\mathrm{Cs}_{2} \mathrm{CO}_{3}$ (2.0 equiv.), and DMSO $(2.0 \mathrm{~mL})$ under $34 \mathrm{~W}$ CFL irradiation in $\mathrm{Ar}$ for $24 \mathrm{~h}$; isolated yield of the product. ${ }^{b} \mathrm{In} \mathrm{MeCN}(2.0 \mathrm{~mL})$.

sequent reduction of alkyl radical $\mathbf{B}$ by $4 \mathrm{CzIPN}^{-}$would generate carbanion $\mathbf{E}$. Deuteration of $\mathbf{E}$ with $\mathrm{D}_{2} \mathrm{O}$ produces the target product 2a (path A). In presence of air, reduction of $\mathrm{O}_{2}$ by $4 \mathrm{CzIPN}^{-}$would generate $\mathrm{O}_{2}{ }^{--}$active species, which couples with alkyl radical $\mathbf{B}$ to generate compound C. Then, $\mathbf{C}$ will abstract protons to produce $\mathbf{D}$, which is further decomposed to ketone 3a (path B).

\section{Conclusion}

A practical and precise decarboxylation processes for both monodeuteratation and oxygenation have been developed. Mono-deuterated organic molecules with various structures were obtained from commercially available carboxylic acids, and ketone products were produced under similar reaction systems under air. These protocols would be viable to medicines as well.

\section{Experimental section}

Unless otherwise noted, all reagents were obtained from 
Table 3 Oxygenation of phenylacetic acid derivatives ${ }^{a}$

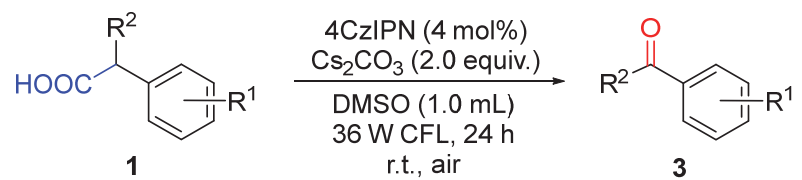

(a) Secondary acid<smiles>COc1ccc2cc(C(C)=O)ccc2c1</smiles>

$3 a, 50 \%$<smiles>O=c1c2ccccc2oc2ccccc12</smiles>

3d, $53 \%$

(c) Complex acid<smiles>CC(=O)c1cccc(C(=O)c2ccccc2)c1</smiles>

$3 g, 19 \%$ (From ketoprofen)<smiles>CC(=O)Nc1ccc(C=O)cc1</smiles>

3e, $19 \%$

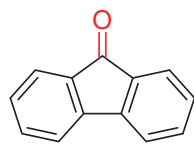

3c, $20 \%$

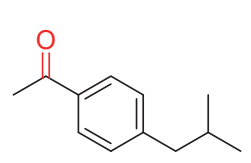

$3 \mathrm{~h}, 18 \%$
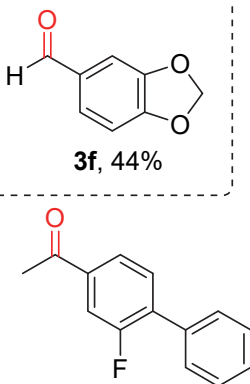

$3 i, 11 \%$<smiles>O=Cc1ccc2c(c1)C(=O)c1ccccc1CO2</smiles>

3j, $15 \%$
(From isoxepac)<smiles>COc1ccc2[nH]c(C)c(C=O)c2c1</smiles>

$3 \mathbf{k}, 21 \%$

(From indometacin)

${ }^{a}$ Reaction conditions: 1 ( $\left.0.1 \mathrm{mmol}\right), 4 \mathrm{CzIPN}$ ( $\left.4 \mathrm{~mol} \%\right), \mathrm{Cs}_{2} \mathrm{CO}_{3}$ (2.0 equiv.), and DMSO $(1.0 \mathrm{~mL})$ under $34 \mathrm{~W}$ CFL irradiation in air for $24 \mathrm{~h}$; isolated yield of the product.

commercial suppliers and used without further purification. The reaction product was isolated by column chromatography on a silica gel (236 400 mesh) column using petroleum ether $(\mathrm{PE})$ with a boiling range from $60{ }^{\circ} \mathrm{C}$ to $90{ }^{\circ} \mathrm{C}$ and EtOAc. ${ }^{1} \mathrm{H}$ NMR, ${ }^{13} \mathrm{C}$ NMR and ${ }^{19} \mathrm{~F}$ NMR spectra were recorded on 400, 101, $376 \mathrm{MHz}$ NMR spectrometers using $\mathrm{CDCl}_{3}$ as solvent. In addition, ${ }^{1} \mathrm{H} \mathrm{NMR}$ and ${ }^{13} \mathrm{C}$ NMR spectra used tetramethylsilane as internal standard and ${ }^{19} \mathrm{~F}$ NMR spectra used trifluoroacetic acid as internal standard. HRMS were made by means of ESI. Unless otherwise noted, all reagents were weighed and handled in air.

\subsection{General procedure for synthesis of deuterated products}

Carboxylic acids (1) (0.1 mmol), $\mathrm{D}_{2} \mathrm{O}$ (3.0 mmol, 0.054 $\mathrm{mL}$ ), $4 \mathrm{CzIPN}$ (0.04 mmol, $3.2 \mathrm{mg}), \mathrm{Cs}_{2} \mathrm{CO}_{3}$ (0.2 mmol, 65.2 $\mathrm{mg})$ and DMSO $(2.0 \mathrm{~mL})$ were added to a $10-\mathrm{mL}$ Schlenk tube. The tube was filled with argon and then sealed, and irradiated with $36 \mathrm{~W}$ CFL (approximately $9 \mathrm{~cm}$ away from the light source). After the complete conversion of the substrates (monitored by TLC), the reaction mixture was concentrated, and the residue was purified by silica gel column<smiles>COc1ccc2cc(C(C)C(=O)O)ccc2c1</smiles>

4 CzIPN (4 mol\%)

$\mathrm{Cs}_{2} \mathrm{CO}_{3}$ (2.0 equiv.)

TEMPO (3.0 equiv.)

DMSO (2.0 mL), $36 \mathrm{~W} \mathrm{CFL}$

$\mathrm{D}_{2} \mathrm{O}, 24$ h, r.t., $\mathrm{Ar}$<smiles>[2H]C(C)c1ccc2cc(OC)ccc2c1</smiles>

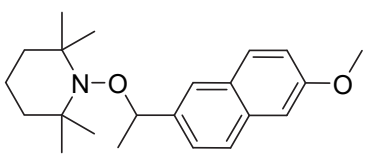

2a, no reaction

4, $35 \%$<smiles>COc1ccc2cc(C(C)C(=O)O)ccc2c1</smiles>

1a<smiles>COc1ccc2cc(C(C)=O)ccc2c1</smiles>

3a, $<5 \%$

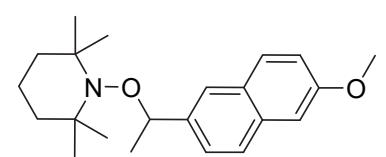

$4,40 \%$

Scheme 2 Mechanism experiment and radical trapping

2a

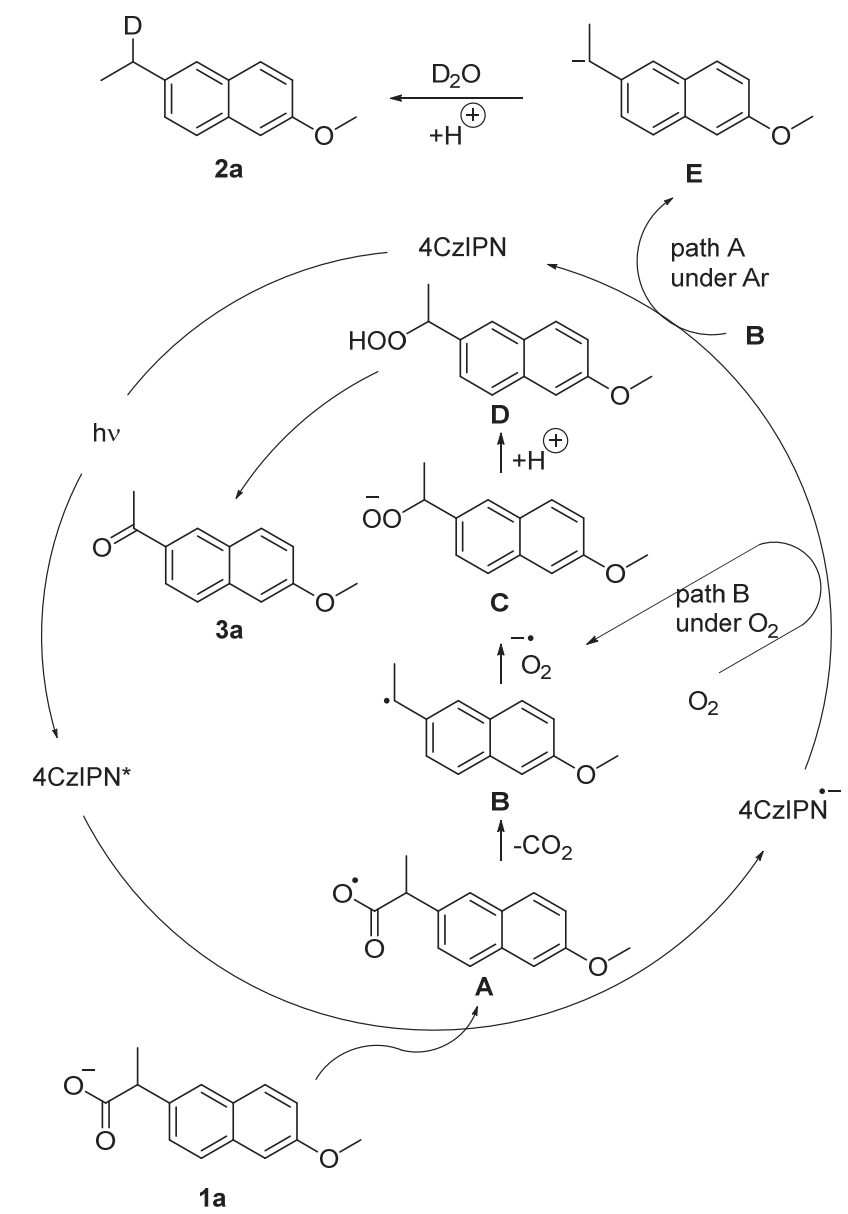

Scheme 3 Proposed reaction pathway

chromatography to give the desired product. 


\subsection{General procedure for synthesis of oxygenation} products

Carboxylic acids (1) $(0.1 \mathrm{mmol}), 4$ CzIPN $(0.04 \mathrm{mmol}$, $3.2 \mathrm{mg}), \mathrm{Cs}_{2} \mathrm{CO}_{3}(0.2 \mathrm{mmol}, 65.2 \mathrm{mg})$ and DMSO $(1.0 \mathrm{~mL})$ were added to a $10 \mathrm{~mL}$ Schlenk tube. The tube was sealed and irradiated with $36 \mathrm{~W}$ CFL (approximately $9 \mathrm{~cm}$ away from the light source). After the complete conversion of the substrates (monitored by TLC), the reaction mixture was concentrated, and the residue was purified by silica gel column chromatography to give the desired product.

\subsection{Mechanism experiment and radical trapping}

A Schlenk tube with a magnetic stir bar was charged with 4CzIPN (0.04 mmol, $3.2 \mathrm{mg}), \mathrm{Cs}_{2} \mathrm{CO}_{3}(0.2 \mathrm{mmol}, 2.0$ equiv.), $\mathrm{D}_{2} \mathrm{O}(3.0 \mathrm{mmol}, 0.054 \mathrm{~mL})$ or without adding $\mathrm{D}_{2} \mathrm{O}$, TEMPO (0.3 mmol, 3.0 equiv.), and substrate 1a (0.1 mmol, 1.0 equiv.) in a Schlenk tube. Then, DMSO was added as solvent. The reaction mixture was stirred for 24 hours under $36 \mathrm{~W}$ LED irradiation. Upon completion, the reaction mixture was concentrated, and the residue was purified by silica gel column chromatography to give the desired product.

2-(Ethyl-1- $d$ )-6-methoxynaphthalene (2a): White solid, (63\% yield, $11.8 \mathrm{mg}$ ). m.p. $48 \sim 49{ }^{\circ} \mathrm{C} ;{ }^{1} \mathrm{H}$ NMR $(400 \mathrm{MHz}$, Chloroform- $d$ ) $\delta: 7.72(\mathrm{dd}, J=8.5,1.9 \mathrm{~Hz}, 2 \mathrm{H}), 7.60(\mathrm{~s}$, $1 \mathrm{H}), 7.37$ (dd, $J=8.4,1.6 \mathrm{~Hz}, 1 \mathrm{H}), 7.19 \sim 7.16(\mathrm{~m}, 2 \mathrm{H})$, $2.86 \sim 2.78(\mathrm{~m}, 1 \mathrm{H}), 1.36(\mathrm{~d}, J=7.5 \mathrm{~Hz}, 3 \mathrm{H}) ;{ }^{13} \mathrm{C}$ NMR (101 MHz, Chloroform- $d$ ) $\delta: 157.1,139.5,133.0,129.2$, $129.0,127.6,126.8,125.5,118.7,105.7,55.3,28.6$ (t, $J=$ 19.2 Hz, C-D), 15.6. HRMS (ESI) calcd for $\mathrm{C}_{13} \mathrm{H}_{14} \mathrm{DO}$ $[\mathrm{M}+\mathrm{H}]^{+}: 188.1180$; found 188.1178 .

(Methylene- $d$ )dibenzene (2b): Yellow oil, 66\% yield, $11.2 \mathrm{mg} .{ }^{1} \mathrm{H}$ NMR $(400 \mathrm{MHz}$, Chloroform- $d$ ) $\delta: 7.38 \sim 7.28$ (m, 4H), $7.23(\mathrm{t}, J=6.2 \mathrm{~Hz}, 6 \mathrm{H}), 4.01(\mathrm{~d}, J=7.9 \mathrm{~Hz}, 1 \mathrm{H})$; ${ }^{13} \mathrm{C}$ NMR (101 MHz, Chloroform- $d$ ) $\delta: 141.13,128.98$, 128.51, 126.12, 41.7 (t, $J=20.2 \mathrm{~Hz}, \mathrm{C}-\mathrm{D})$. HRMS (ESI) calcd for $\mathrm{C}_{13} \mathrm{H}_{12} \mathrm{D}[\mathrm{M}+\mathrm{H}]^{+}: 170.1075$; found 170.1074 .

9H-Fluorene-9-d (2c): White solid, 77\% yield, $12.9 \mathrm{mg}$. m.p. $108 \sim 109{ }^{\circ} \mathrm{C}$; ${ }^{1} \mathrm{H}$ NMR (400 MHz, Chloroform- $d$ ) $\delta$ : $7.82(\mathrm{~d}, J=7.5 \mathrm{~Hz}, 2 \mathrm{H}), 7.57(\mathrm{~d}, J=7.3 \mathrm{~Hz}, 2 \mathrm{H}), 7.40(\mathrm{t}$, $J=7.4 \mathrm{~Hz}, 2 \mathrm{H}), 7.33(\mathrm{t}, J=7.3 \mathrm{~Hz}, 2 \mathrm{H}), 3.92(\mathrm{~d}, J=8.2 \mathrm{~Hz}$, $1 \mathrm{H}) ;{ }^{13} \mathrm{C}$ NMR (101 MHz, Chloroform- $d$ ) $\delta: 143.2,141.8$, 126.8, 125.1, 119.9, 36.7 (t, $J=20.2 \mathrm{~Hz}, \mathrm{C}-\mathrm{D})$. HRMS (ESI) calcd for $\mathrm{C}_{13} \mathrm{H}_{10} \mathrm{D}[\mathrm{M}+\mathrm{H}]^{+}$168.0918; found 168.0916.

$9 H$-Xanthene-9- $d$ (2d): White solid, 76\% yield, $13.9 \mathrm{mg}$. m.p. $89 \sim 90{ }^{\circ} \mathrm{C} ;{ }^{1} \mathrm{H}$ NMR $(400 \mathrm{MHz}$, Chloroform- $d) \delta$ : $7.29 \sim 7.15(\mathrm{~m}, 4 \mathrm{H}), 7.13 \sim 7.01(\mathrm{~m}, 4 \mathrm{H}), 4.06(\mathrm{~d}, J=7.9$ $\mathrm{Hz}, 1 \mathrm{H}) ;{ }^{13} \mathrm{C}$ NMR (101 MHz, Chloroform- $d$ ) $\delta: 152.0$, 129.0, 127.7, 123.0, 120.6, 116.5, $27.6(\mathrm{t}, J=20.2 \mathrm{~Hz}$, C-D). HRMS (ESI) calcd for $\mathrm{C}_{13} \mathrm{H}_{10} \mathrm{DO}[\mathrm{M}+\mathrm{H}]^{+}$ 184.0867; found 184.0865 .

$N$-(4-(Methyl- $d$ )phenyl)acetamide (2e): White solid, $17 \%$ yield, 2.5 mg. m.p. $142 \sim 143{ }^{\circ} \mathrm{C} ;{ }^{1} \mathrm{H}$ NMR $(400 \mathrm{MHz}$, Chloroform- $d$ ) $\delta: 7.37$ (d, $J=8.3 \mathrm{~Hz}, 2 \mathrm{H}), 7.11$ (d, $J=8.2$ $\mathrm{Hz}, 3 \mathrm{H}), 2.30$ (d, $J=6.1 \mathrm{~Hz}, 2 \mathrm{H}), 2.16(\mathrm{~s}, 3 \mathrm{H}) ;{ }^{13} \mathrm{C}$ NMR (101 MHz, Chloroform-d) $\delta: 168.2,135.3,133.9,129.5$,
120.0, 24.6, 20.6 (t, $J=19.2 \mathrm{~Hz}, \mathrm{C}-\mathrm{D})$. HRMS (ESI) calcd for $\mathrm{C}_{9} \mathrm{H}_{11} \mathrm{DNO}[\mathrm{M}+\mathrm{H}]^{+}$151.0976; found 151.0974.

4-(Methyl- $d$ )-1,1'-biphenyl (2f): White solid, $11 \%$ yield, 1.9 mg. m.p. $39 \sim 40{ }^{\circ} \mathrm{C} ;{ }^{1} \mathrm{H}$ NMR $(400 \mathrm{MHz}$, Chloroform-d) $\delta: 7.62 \sim 7.56(\mathrm{~m}, 2 \mathrm{H}), 7.50(\mathrm{~d}, J=8.1 \mathrm{~Hz}, 2 \mathrm{H})$, $7.43(\mathrm{t}, J=7.6 \mathrm{~Hz}, 2 \mathrm{H}), 7.33(\mathrm{tt}, J=6.7,1.1 \mathrm{~Hz}, 1 \mathrm{H}), 7.26$ $(\mathrm{d}, J=8.2 \mathrm{~Hz}, 2 \mathrm{H}), 2.40 \sim 2.38(\mathrm{~m}, 2 \mathrm{H}) ;{ }^{13} \mathrm{C}$ NMR $(101$ $\mathrm{MHz}$, Chloroform-d) $\delta: 141.2,138.4,137.0,137.0,129.5$, 128.7, 127.0, 127.0, 20.8 (t, $J=19.2 \mathrm{~Hz}, \mathrm{C}-\mathrm{D})$. HRMS (ESI) calcd for $\mathrm{C}_{13} \mathrm{H}_{12} \mathrm{D}[\mathrm{M}+\mathrm{H}]^{+}$170.1075; found 170.1073 .

(3-(Ethyl-1-d)phenyl)(phenyl)methanone (2g): Yellow oil, $83 \%$ yield, $17.5 \mathrm{mg} .{ }^{1} \mathrm{H}$ NMR $(400 \mathrm{MHz}$, Chloroform- $d$ ) $\delta: 7.83 \sim 7.78(\mathrm{~m}, 2 \mathrm{H}), 7.66(\mathrm{~s}, 1 \mathrm{H}), 7.63 \sim 7.56(\mathrm{~m}$, $2 \mathrm{H}), 7.48(\mathrm{t}, J=7.6 \mathrm{~Hz}, 2 \mathrm{H}), 7.40(\mathrm{dt}, J=14.9,7.6 \mathrm{~Hz}, 2 \mathrm{H})$, $2.75 \sim 2.67(\mathrm{~m}, 1 \mathrm{H}), 1.26(\mathrm{~d}, J=7.5 \mathrm{~Hz}, 3 \mathrm{H}) ;{ }^{13} \mathrm{C} \mathrm{NMR}$ (101 MHz, Chloroform- $d$ ) $\delta:$ 197.1, 144.5, 137.8, 137.7, $132.3,132.1,130.1,129.4,128.3,128.2,127.7,28.4$ (t, $J=$ $20.2 \mathrm{~Hz}, \mathrm{C}-\mathrm{D}$ ), 15.5. HRMS (ESI) calcd for $\mathrm{C}_{15} \mathrm{H}_{14} \mathrm{DO}$ $[\mathrm{M}+\mathrm{H}]^{+}$212.1180; found 212.1178.

6-Chloro-2-(ethyl-1-d)-9H-carbazole (2h): White solid, $13 \%$ yield, 3.0 mg. m.p. $201 \sim 202{ }^{\circ} \mathrm{C} ;{ }^{1} \mathrm{H}$ NMR $(400 \mathrm{MHz}$, Chloroform- $d$ ) $\delta: 8.03(\mathrm{~d}, J=7.7 \mathrm{~Hz}, 1 \mathrm{H}), 7.97(\mathrm{~d}, \quad J=8.0$ $\mathrm{Hz}, 2 \mathrm{H}), 7.45 \sim 7.34$ (m, 2H), 7.21 (ddd, $J=8.0,6.6,1.6$ $\mathrm{Hz}, 1 \mathrm{H}), 7.10 \sim 7.08(\mathrm{~m}, 1 \mathrm{H}), 2.85 \sim 2.77(\mathrm{~m}, 1 \mathrm{H}), 1.32(\mathrm{~d}$, $J=7.6 \mathrm{~Hz}, 3 \mathrm{H}) ;{ }^{13} \mathrm{C}$ NMR $(101 \mathrm{MHz}$, Chloroform- $d) \delta$ : $142.6,140.0,139.6,125.3,123.5,121.3,120.1,120.0$, 119.9, 119.3, 110.4, 109.5, 29.1 (t, $J=20.2 \mathrm{~Hz}, \mathrm{C}-\mathrm{D}), 16.0$. HRMS (ESI) calcd for $\mathrm{C}_{14} \mathrm{H}_{12} \mathrm{DClN}[\mathrm{M}+\mathrm{H}]^{+}$231.0794; found 231.0792 .

2-(Methyl- $d$ )dibenzo[b,e]oxepin-11(6H)-one (2i): White solid, 50\% yield, $11.3 \mathrm{mg}$. m.p. $71 \sim 72{ }^{\circ} \mathrm{C}$; ${ }^{1} \mathrm{H}$ NMR $(400$ MHz, Chloroform- $d$ ) $\delta: 8.03(\mathrm{~s}, 1 \mathrm{H}), 7.90(\mathrm{~d}, J=7.6 \mathrm{~Hz}$, $1 \mathrm{H}), 7.54$ (t, $J=7.2 \mathrm{~Hz}, 1 \mathrm{H}), 7.46$ (t, $J=7.4 \mathrm{~Hz}, 1 \mathrm{H}), 7.35$ $(\mathrm{d}, J=7.4 \mathrm{~Hz}, 1 \mathrm{H}), 7.29(\mathrm{~d}, J=8.3 \mathrm{~Hz}, 1 \mathrm{H}), 6.95(\mathrm{~d}, J=8.3$ $\mathrm{Hz}, 1 \mathrm{H}), 5.16$ (s, 2H), 2.34 (s, 2H); ${ }^{13} \mathrm{C}$ NMR $(101 \mathrm{MHz}$, Chloroform- $d$ ) $\delta:$ 191.2, 159.4, 140.6, 136.4, 135.8, 132.6, $131.6,131.5,129.5,129.2,127.7,125.1,120.5,73.7,20.1$ (t, $J=19.2 \mathrm{~Hz}, \mathrm{C}-\mathrm{D})$. HRMS (ESI) calcd for $\mathrm{C}_{15} \mathrm{H}_{12} \mathrm{DO}_{2}$ $[\mathrm{M}+\mathrm{H}]^{+}$226.0973; found 226.0971.

(4-Chlorophenyl)(5-methoxy-2-methyl-3-(methyl- $d$ )$1 H$-indol-1-yl)methanone (2j): Yellow oil, $11 \%$ yield, 3.5 mg. ${ }^{1} \mathrm{H}$ NMR (400 MHz, Chloroform- $d$ ) $\delta: 7.64$ (d, $J=8.5$ $\mathrm{Hz}, 2 \mathrm{H}), 7.46(\mathrm{~d}, J=8.5 \mathrm{~Hz}, 2 \mathrm{H}), 6.96 \sim 6.85(\mathrm{~m}, 2 \mathrm{H}), 6.66$ $(\mathrm{dd}, J=8.9,2.6 \mathrm{~Hz}, 1 \mathrm{H}), 3.85(\mathrm{~s}, 3 \mathrm{H}), 2.31(\mathrm{~s}, 3 \mathrm{H}), 2.17(\mathrm{~s}$, $2 \mathrm{H}) ;{ }^{13} \mathrm{C}$ NMR (101 MHz, Chloroform- $d$ ) $\delta: 168.2,156.0$, $138.9,134.4,133.8,132.0,131.0,130.9,129.0,115.4$, $114.9,111.2,101.3,55.7,13.3,8.5$ (t, $J=19.2 \mathrm{~Hz}, \mathrm{C}-\mathrm{D})$. HRMS (ESI) calcd for $\mathrm{C}_{18} \mathrm{H}_{16} \mathrm{DClNO}_{2}[\mathrm{M}+\mathrm{H}]$ 315.1005; found 315.1003.

1-(6-Methoxynaphthalen-2-yl)ethan-1-one (3a): White solid, $50 \%$ yield, 10.0 mg. m.p. $107 \sim 108{ }^{\circ} \mathrm{C} ;{ }^{1} \mathrm{H}$ NMR $(400 \mathrm{MHz}$, Chloroform- $d$ ) $\delta: 8.40$ (s, 1H), 8.01 (dd, $J=8.7$, $1.5 \mathrm{~Hz}, 1 \mathrm{H}), 7.86(\mathrm{~d}, J=8.9 \mathrm{~Hz}, 1 \mathrm{H}), 7.77(\mathrm{~d}, J=8.7 \mathrm{~Hz}$, $1 \mathrm{H}), 7.21(\mathrm{dd}, J=8.9,2.4 \mathrm{~Hz}, 1 \mathrm{H}), 7.16(\mathrm{~s}, 1 \mathrm{H}), 3.95$ (s, $3 \mathrm{H}), 2.70(\mathrm{~s}, 3 \mathrm{H}) ;{ }^{13} \mathrm{C}$ NMR (101 MHz, Chloroform- $d$ ) $\delta$ : $197.9,159.8,137.3,132.6,131.1,130.1,127.8,127.1$, 
124.7, 119.8, 105.8, 55.4, 26.6. HRMS (ESI) calcd for $\mathrm{C}_{13} \mathrm{H}_{13} \mathrm{O}_{2}[\mathrm{M}+\mathrm{H}]^{+}$201.0910; found 201.0910.

Benzophenone (3b): White solid, 13\% yield, $2.4 \mathrm{mg}$. m.p. $47 \sim 48{ }^{\circ} \mathrm{C} ;{ }^{1} \mathrm{H}$ NMR (400 MHz, Chloroform-d) $\delta$ : $7.88 \sim 7.76(\mathrm{~m}, 4 \mathrm{H}), 7.62 \sim 7.58(\mathrm{~m}, 2 \mathrm{H}), 7.49(\mathrm{t}, J=7.6$ $\mathrm{Hz}, 4 \mathrm{H}) ;{ }^{13} \mathrm{C}$ NMR (101 MHz, Chloroform- $d$ ) $\delta: 196.8$, 137.6, 132.5, 130.1, 128.3. HRMS (ESI) calcd for $\mathrm{C}_{13} \mathrm{H}_{11} \mathrm{O}[\mathrm{M}+\mathrm{H}]^{+}$183.0804; found 183.0802.

9H-Fluoren-9-one (3c): Yellow solid, 20\% yield, $3.6 \mathrm{mg}$. m.p. $80 \sim 81{ }^{\circ} \mathrm{C} ;{ }^{1} \mathrm{H}$ NMR (400 MHz, Chloroform- $d$ ) $\delta$ : $7.66(\mathrm{~d}, J=7.4 \mathrm{~Hz}, 2 \mathrm{H}), 7.52 \sim 7.46(\mathrm{~m}, 4 \mathrm{H}), 7.29(\mathrm{td}, J=$ 7.2, $1.4 \mathrm{~Hz}, 2 \mathrm{H}) ;{ }^{13} \mathrm{C}$ NMR (101 MHz, Chloroform-d) $\delta$ : $193.9,144.4,134.7,134.2,129.1,124.3,120.3$. HRMS (ESI) calcd for $\mathrm{C}_{13} \mathrm{H}_{9} \mathrm{O}[\mathrm{M}+\mathrm{H}]^{+}$181.0648; found 181.0646.

9H-Xanthen-9-one (3d): White solid, 53\% yield, 10.4 mg. m.p. $172 \sim 173{ }^{\circ} \mathrm{C} ;{ }^{1} \mathrm{H} \mathrm{NMR}(400 \mathrm{MHz}$, Chloroform- $d$ ) $\delta: 8.36(\mathrm{dd}, J=7.9,1.6 \mathrm{~Hz}, 2 \mathrm{H}), 7.78 \sim 7.68(\mathrm{~m}, 2 \mathrm{H}), 7.51$ $(\mathrm{d}, J=8.4 \mathrm{~Hz}, 2 \mathrm{H}), 7.39(\mathrm{t}, J=7.2 \mathrm{~Hz}, 2 \mathrm{H}) ;{ }^{13} \mathrm{C}$ NMR $(101$ $\mathrm{MHz}$, Chloroform-d) $\delta: 177.2,156.2,134.8,126.7,123.9$, 121.9, 118.0. HRMS (ESI) calcd for $\mathrm{C}_{13} \mathrm{H}_{9} \mathrm{O}_{2}[\mathrm{M}+\mathrm{H}]^{+}$ 197.0597; found 197.0595.

$\mathrm{N}$-(4-Formylphenyl)acetamide (3e): White solid, 19\% yield, $3.1 \mathrm{mg}$. m.p. $144 \sim 145{ }^{\circ} \mathrm{C}$; ${ }^{1} \mathrm{H}$ NMR $(400 \mathrm{MHz}$, Chloroform-d) $\delta: 9.94(\mathrm{~s}, 1 \mathrm{H}), 7.87$ (d, $J=8.6 \mathrm{~Hz}, 2 \mathrm{H})$, $7.71(\mathrm{~d}, J=8.4 \mathrm{~Hz}, 2 \mathrm{H}), 7.44(\mathrm{~s}, 1 \mathrm{H}), 2.25(\mathrm{~s}, 3 \mathrm{H}) ;{ }^{13} \mathrm{C}$ NMR (101 MHz, Chloroform- $d$ ) $\delta$ : 191.0, 168.5, 143.4, 132.4, 131.2, 119.2, 24.8. HRMS (ESI) calcd for $\mathrm{C}_{9} \mathrm{H}_{10} \mathrm{NO}_{2}[\mathrm{M}+\mathrm{H}]^{+}$164.0706; found 164.0704.

Benzo $[d][1,3]$ dioxole-5-carbaldehyde (3f): White solid, $44 \%$ yield, 6.6 mg. m.p. $36 \sim 37{ }^{\circ} \mathrm{C} ;{ }^{1} \mathrm{H}$ NMR $(400 \mathrm{MHz}$, Chloroform- $d$ ) $\delta: 9.81(\mathrm{~s}, 1 \mathrm{H}), 7.41$ (dd, $J=7.9,1.4 \mathrm{~Hz}$, 1H), 7.34 (d, $J=1.3 \mathrm{~Hz}, 1 \mathrm{H}), 6.93$ (d, $J=7.9 \mathrm{~Hz}, 1 \mathrm{H}), 6.08$ (s, 2H); ${ }^{13} \mathrm{C}$ NMR (101 MHz, Chloroform-d) $\delta$ : 190.3, $153.1,148.7,131.9,128.7,108.4,106.9,102.1$. HRMS (ESI) calcd for $\mathrm{C}_{8} \mathrm{H}_{7} \mathrm{O}_{3}[\mathrm{M}+\mathrm{H}]^{+}$151.0390; found 151.0389 .

1-(3-Benzoylphenyl)ethan-1-one (3g): Colorless oil, $19 \%$ yield, $4.3 \mathrm{mg} .{ }^{1} \mathrm{H}$ NMR (400 MHz, Chloroform- $d$ ) $\delta$ : 8.37 (s, 1H), 8.19 (d, $J=7.8 \mathrm{~Hz}, 1 \mathrm{H}), 7.99$ (d, $J=7.7 \mathrm{~Hz}$, $1 \mathrm{H}), 7.80$ (d, $J=7.2 \mathrm{~Hz}, 2 \mathrm{H}), 7.62$ (q, $J=7.8 \mathrm{~Hz}, 2 \mathrm{H}), 7.51$ $(\mathrm{t}, \quad J=7.6 \mathrm{~Hz}, 2 \mathrm{H}), 2.65(\mathrm{~s}, 3 \mathrm{H}) ;{ }^{13} \mathrm{C} \mathrm{NMR}(101 \mathrm{MHz}$, Chloroform- $d$ ) $\delta$ : 197.4, 195.9, 138.1, 137.2, 137.0, 134.3, 132.9, 131.8, 130.1, 129.7, 128.8, 128.5, 26.8. HRMS (ESI) calcd for $\mathrm{C}_{15} \mathrm{H}_{13} \mathrm{O}_{2}[\mathrm{M}+\mathrm{H}]^{+}$225.0910; found 225.0910.

1-(4-Isobutylphenyl)ethan-1-one (3h): Colorless oil, $18 \%$ yield, $3.2 \mathrm{mg} .{ }^{1} \mathrm{H}$ NMR (400 MHz, Chloroform- $d$ ) $\delta$ : 7.87 (d, $J=8.2 \mathrm{~Hz}, 2 \mathrm{H}), 7.23$ (d, $J=8.3 \mathrm{~Hz}, 2 \mathrm{H}), 2.58$ (s, $3 \mathrm{H}), 2.53(\mathrm{~d}, J=7.2 \mathrm{~Hz}, 2 \mathrm{H}), 1.90(\mathrm{dp}, J=13.5,6.6 \mathrm{~Hz}$, $1 \mathrm{H}), 0.91$ (d, $J=6.6 \mathrm{~Hz}, 6 \mathrm{H}) ;{ }^{13} \mathrm{C}$ NMR $(101 \mathrm{MHz}$, Chloroform- $d$ ) $\delta$ : 198.0, 147.6, 135.0, 129.3, 128.3, 45.4, 30.1, 26.6, 22.3. HRMS (ESI) calcd for $\mathrm{C}_{12} \mathrm{H}_{17} \mathrm{O}[\mathrm{M}+\mathrm{H}]^{+}$ 177.1274; found 177.1272 .

1-(2-Fluoro-[1,1'-biphenyl]-4-yl)ethan-1-one (3i): White solid, $11 \%$ yield, 2.4 mg. m.p. 94 95 ${ }^{\circ} \mathrm{C} ;{ }^{1} \mathrm{H}$ NMR (400 MHz, Chloroform- $d$ ) $\delta: 7.81$ (dd, $J=8.0,1.5 \mathrm{~Hz}, 1 \mathrm{H}), 7.74$ $(\mathrm{dd}, J=11.2,1.4 \mathrm{~Hz}, 1 \mathrm{H}), 7.56(\mathrm{q}, J=8.2 \mathrm{~Hz}, 3 \mathrm{H}), 7.45$ (dt,
$J=23.0,7.2 \mathrm{~Hz}, 3 \mathrm{H}), 2.63(\mathrm{~s}, 3 \mathrm{H}) ;{ }^{13} \mathrm{C} \mathrm{NMR}(101 \mathrm{MHz}$, Chloroform- $d$ ) $\delta$ : 196.5, 159.7 (d, $J=249.9 \mathrm{~Hz}), 137.8(\mathrm{~d}$, $J=6.4 \mathrm{~Hz}), 134.7$ (d, $J=1.2 \mathrm{~Hz}), 133.8(\mathrm{~d}, J=13.6 \mathrm{~Hz})$, 130.9 (d, $J=3.4 \mathrm{~Hz}), 129.0$ (d, $J=3.1 \mathrm{~Hz}), 128.6,128.5$, 124.3 (d, $J=3.4 \mathrm{~Hz}), 115.9$ (d, $J=24.0 \mathrm{~Hz}), 26.7 ;{ }^{19} \mathrm{~F} \mathrm{NMR}$ (376 MHz, Chloroform- $d$ ) $\delta$ : -40.68 . HRMS (ESI) calcd for $\mathrm{C}_{14} \mathrm{H}_{12} \mathrm{FO}[\mathrm{M}+\mathrm{H}]^{+}$215.0867; found 215.0865.

2-Acetyldibenzo[b,e]oxepin-11(6H)-one (3j): Colorless oil, $15 \%$ yield, $3.8 \mathrm{mg} .{ }^{1} \mathrm{H}$ NMR (400 MHz, Chloroform- $d$ ) $\delta: 10.01(\mathrm{~s}, 1 \mathrm{H}), 8.74(\mathrm{~s}, 1 \mathrm{H}), 8.03(\mathrm{~d}, J=8.4 \mathrm{~Hz}, 1 \mathrm{H}), 7.89$ $(\mathrm{d}, J=7.4 \mathrm{~Hz}, 1 \mathrm{H}), 7.61(\mathrm{t}, J=7.5 \mathrm{~Hz}, 1 \mathrm{H}), 7.52(\mathrm{t}, J=7.6$ $\mathrm{Hz}, 1 \mathrm{H}), 7.41$ (d, $J=7.3 \mathrm{~Hz}, 1 \mathrm{H}), 7.18(\mathrm{~d}, J=8.6 \mathrm{~Hz}, 1 \mathrm{H})$, $5.29(\mathrm{~s}, 2 \mathrm{H}) ;{ }^{13} \mathrm{C}$ NMR (101 MHz, Chloroform-d) $\delta: 190.4$, $190.2,165.6,140.4,137.5,134.5,133.4,133.2,131.0$, 129.8, 129.4, 128.2, 125.1, 122.2, 73.6. HRMS (ESI) calcd for $\mathrm{C}_{16} \mathrm{H}_{13} \mathrm{O}_{3}[\mathrm{M}+\mathrm{H}]^{+}$253.0859; found 253.0857.

5-Methoxy-2-methyl-1 $H$-indole-3-carbaldehyde (3k): White solid, $21 \%$ yield, 4.0 mg. m.p. $173 \sim 174{ }^{\circ} \mathrm{C} ;{ }^{1} \mathrm{H}$ NMR (400 MHz, Chloroform- $d$ ) $\delta: 10.16(\mathrm{~s}, 1 \mathrm{H}), 8.52$ (s, $1 \mathrm{H}), 7.78(\mathrm{~s}, 1 \mathrm{H}), 7.22$ (d, $J=8.8 \mathrm{~Hz}, 1 \mathrm{H}), 6.88$ (dd, $J=$ 8.8, $2.4 \mathrm{~Hz}, 1 \mathrm{H}), 3.89$ (s, 3H), 2.73 (s, 3H); ${ }^{13} \mathrm{C} \mathrm{NMR}(101$ $\mathrm{MHz}$, Chloroform- $d$ ) $\delta: 184.5,156.5,146.9,129.6,126.8$, 114.9, 113.5, 111.4, 102.8, 55.8, 12.2. HRMS (ESI) calcd for $\mathrm{C}_{11} \mathrm{H}_{12} \mathrm{NO}_{2}[\mathrm{M}+\mathrm{H}]^{+}$190.2215; found 190.2213.

1-(1-(6-Methoxynaphthalen-2-yl)ethoxy)-2,2,6,6-tetramethylpiperidine (4): White solid, 40\% yield, $13.6 \mathrm{mg}$. m.p. $101 \sim 102{ }^{\circ} \mathrm{C} ;{ }^{1} \mathrm{H}$ NMR (400 MHz, Chloroform-d) $\delta$ : $7.73 \sim 7.69(\mathrm{~m}, 2 \mathrm{H}), 7.65(\mathrm{~s}, 1 \mathrm{H}), 7.49 \sim 7.46(\mathrm{~m}, 1 \mathrm{H})$, $7.15 \sim 7.12(\mathrm{~m}, 2 \mathrm{H}), 4.91(\mathrm{q}, J=6.6 \mathrm{~Hz}, 1 \mathrm{H}), 3.92(\mathrm{~s}, 3 \mathrm{H})$, $1.66 \sim 0.91(\mathrm{~m}, 18 \mathrm{H}), 0.62(\mathrm{~s}, 3 \mathrm{H}) ;{ }^{13} \mathrm{C}$ NMR $(101 \mathrm{MHz}$, Chloroform- $d$ ) $\delta$ : 157.4, 141.1, 133.7, 129.4, 128.7, 126.6, 125.8, 125.0, 118.6, 105.7, 83.3, 59.9, 59.6, 55.3, 40.4, 34.4, 23.5, 20.3, 17.2. HRMS (ESI) calcd for $\mathrm{C}_{22} \mathrm{H}_{32} \mathrm{NO}_{2}[\mathrm{M}+$ $\mathrm{H}]^{+}$342.2428; found 342.2426.

Supporting Information Copies of ${ }^{1} \mathrm{H}$ NMR, ${ }^{13} \mathrm{C}$ NMR and ${ }^{19} \mathrm{~F}$ NMR spectra for compounds $\mathbf{2} \mathbf{a} \sim \mathbf{2} \mathbf{j}$ and $\mathbf{3 a} \sim \mathbf{3 k}$. The Supporting Information is available free of charge via the Internet at http://sioc-journal.cn.

\section{References}

[1] (a) Loh, Y. Y.; Nagao, K.; Hoover, A. J.; Hesk, D.; Rivera, N. R.; Colletti, S. L.; Davies, I. W.; MacMillan, D. W. C. Science 2017, $358,1182$.

(b) Atzrodt, J.; Derdau, V.; Kerr, W. J.; Reid, M. Angew. Chem., Int. Ed. 2018, 57, 1758.

[2] (a) Zhang, P.-P.; Huang, D.; Newhouse, T. R. J. Am. Chem. Soc. 2020, 142, 1757.

(b) Sterckx, H.; De Houwer, J.; Mensch, C.; Herrebout, W.; Tehrani, K. A.; Maes, B. U. W. Beilstein J. Org. Chem. 2016, 12, 144. (c) Liu, J.-Z.; Wen, X.-J.; Qin, C.; Li, X.-Y.; Luo, X.; Sun, A.; Zhu, B.-C.; Song, S.; Jiao, N. Angew. Chem., Int. Ed. 2017, 56, 11940.

[3] (a) Atzrodt, J.; Derdau, V.; Kerr, W. J.; Reid, M. Angew. Chem., Int. Ed. 2018, 57, 3022.

(b) Atzrodt, J.; Derdau, V.; Fey, T.; Zimmermann, J. Angew. Chem., Int. Ed. 2007, 46, 7744.

(c) Bai, W.; Lee, K.-H.; Tse, S. K. S.; Chan, K. W.; Lin, Z.-Y.; Jia, G.-C. Organometallics 2015, 34, 3686.

(d) Tse, S. K. S.; Xue, P.; Lau, C. W. S.; Sung, H. H. Y.; Williams, I. 
D.; Jia, G.-C. Chem.-Eur. J. 2011, 17, 13918.

(e) Neubert, L.; Michalik, D.; Bähn, S.; Imm, S.; Neumann, H.; Atzrodt, J.; Derdau, V.; Holla, W.; Beller, M. J. Am. Chem. Soc. 2012, 134, 12239.

(f) Hale, L. V. A.; Szymczak, N. K. J. Am. Chem. Soc. 2016, 138, 13489.

[4] Modvig, A.; Andersen, T. L.; Taaning, R. H.; Lindhardt, A. T.; Skrydstrup, T. J. Org. Chem. 2014, 79, 5861.

[5] (a) Cook, A.; Prakash, S.; Zheng, Y.-L.; Newman, S. G. J. Am. Chem. Soc. 2020, 142, 8109.

(b) Eisele, P.; Ullwer, F.; Scholz, S.; Plietker, B. Chem.-Eur. J. 2019, $25,16550$.

(c) Li, H.-Z.; Hou, Y.-X.; Liu, C.-W.; Lai, Z.-M.; Ning, L.; Szostak, R.; Szostak, M.; An, J. Org. Lett. 2020, 22, 1249.

(d) Zhu, N.-B.; Su, M.; Wan, W.-M.; Li, Y.-J.; Bao, H.-L. Org. Lett. 2020, 22, 991.

(e) Ding, Y.-X.; Luo, S.-H.; Weng, C.-Q.; An, J. J. Org. Chem. 2019, 84, 15098.

(f) Li, H.-Z.; Zhang, B.; Dong, Y.-H.; Liu, T.; Zhang, Y.-T.; Nie, H.-Y.; Yang, R.-Y.; Ma, X.-D.; Ling, Y.; An, J. Tetrahedron Lett. 2017, 58, 2757.

[6] (a) Gallezot, P. Chem. Soc. Rev. 2012, 41, 1538.

(b) Rodríguez, N.; Goossen, L. J. Chem. Soc. Rev. 2011, 40, 5030.

(c) Xie, L.-Y.; Bai, Y.-S.; Xu, X.-Q.; Peng, X.; Tang, H.-S.; Huang, Y.; Lin, Y.-W.; Cao, Z.; He, W.-M. Green Chem. 2020, 22, 1720.

(d) Xie, L.-Y.; Peng, S.; Yang, L.-H.; Peng, C.; Lin, Y.-W.; Yu, X.; Cao, Z.; Peng, Y.-Y.; He, W.-M. Green Chem. 2021, 23, 374.

(e) Zhao, F.; Guo, S.; Zhang, Y.; Sun, T.; Yang, B.; Ye, Y.; Sun, K. Org. Chem. Front. 2021, DOI: 10.1039/d1qo01425k.

(f) Wang, X.; Li, G.-F.; Sun, K.; Zhang, B. Chin. J. Org. Chem. 2020, 40, 913 (in Chinese).

(王薪, 李国锋, 孙凯, 张冰, 有机化学, 2020, 40, 913.)

(g) Sun, K.; Li, G.-F.; Li. Y.-Y.; Yu, J.; Zhao, Q.; Zhang, Z.-G.; Zhang, G.-S. Adv. Synth. Catal. 2020, 362, 1947.

(h) Bi, M.-X.; Qian, P.; Wang, Y.-K.; Zha, Z.-G.; Wang, Z.-Y. Chin. Chem. Lett. 2017, 28, 1159.

[7] (a) Liu, C.-B.; Chen, Z.-X.; Su, C.-L.; Zhao, X.-X.; Gao, Q.; Ning, G.-H.; Zhu, H.; Tang, W.; Leng, K.; Fu, W.; Tian, B.-B.; Peng, X.-W.; Li, J.; Xu, Q.-H.; Zhou, W.; Loh, K. P. Nat. Commun. 2018 , 9,80 .

(b) Liu, C.-B.; Han, S.-Y.; Li, M.-Y.; Chong, X.-D.; Zhang, B. Angew. Chem., Int. Ed. 2020, 59, 18527.

(c) Dong, Y.-Y.; Su, Y.-L.; Du, L.-L.; Wang, R.-F.; Zhang, L.; Zhao, D.-B.; Xie, W. ACS Nano 2019, 13, 10754.

[8] Matsubara, S.; Yokota, Y.; Oshima, K. Org. Lett. 2004, 6, 2071.

[9] Rudzki, M.; Alcalde-Aragonés, A.; Dzik, W. I.; Rodríguez, N.; Gooßen, L. J. Synthesis 2012, 44, 184.

[10] (a) Narayanam, J. M. R.; Stephenson, C. R. J. Chem. Soc. Rev. 2011, 40, 102 .

(b) Xuan, J.; Xiao, W.-J. Angew. Chem., Int. Ed. 2012, 51, 6828.

(c) He, W.-M.; Cui, X.-L. Chin. Chem. Lett. 2021, 32, 1589. (d) Zhao, Q.-Q.; Chen, J.-R. Chin. J. Org. Chem. 2021, 41, 871 (in Chinese).

(赵全庆, 陈加荣, 有机化学, 2021, 41, 871.)

(e) Liu, X.; Li, W.; Zhuang, C.-Z.; Cao, H. Chin. J. Org. Chem. 2021, 41, 3459 (in Chinese)

(刘想, 李文, 庄灿展, 曹华, 有机化学, 2021, 41, 3459.)

(f) Wang, X.; Zhang, Y.; Sun, K.; Meng, J.-P.; Zhang, B. Chin. J. Org. Chem. 2021, 41,4588 (in Chinese).

(王薪, 张艳, 孙凯, 孟建萍, 张冰, 有机化学, 2021, 41, 4588.)

(g) Wang, S.-W.; Yu, J.; Zhou, Q.-Y.; Chen, S.-Y.; Xu, Z.-H.; Tang, S. ACS Sustainable Chem. Eng. 2019, 7, 10154.

(h) Yang, H.-Q.; Chen, Q.-Q.; Liu, F.-L.; Shi, R.; Chen, Y. Chin. Chem. Lett. 2021, 32, 676.

(i) Kong, Y.-L.; Xu, W.-X.; Liu, X.-H.; Weng, J.-Q. Chin. Chem. Lett. 2020, 31, 3245.

(j) Xie, L.-Y.; Liu, Y.-S.; Ding, H.-R.; Gong, S.-F.; Tan, J.-X.; He, J.-Y.; Cao, Z.; He, W.-M. Chin. J. Catal. 2020, 4l, 1168.

(k) Meng, X.-X.; Kang, Q.-Q.; Zhang, J.-Y.; Li, Q.; Wei, W.-T.; He W.-M. Green Chem. 2020, 22, 1388.

(1) Gui, Q.-W.; Teng, F.; Li, Z.-C.; Xiong, Z.-Y.; Jin, X.-F.; Liu, H.-Y.; Lin, Y.-W.; Cao, Z.; He, W.-M. Chin. Chem. Lett. 2021, 32, 1907.

[11] Patra, T.; Mukherjee, S.; Ma, J.; Strieth-Kalthoff, F.; Glorius, F. Angew. Chem., Int. Ed. 2019, 58, 10514.

[12] (a) Li, N.; Ning, Y.-Y.; Wu, X.-P.; Xie, J.; Li, W.-P.; Zhu, C.-J. Chem. Sci. 2021, 12, 5505.

(b) Itou, T.; Yoshimi, Y.; Nishikawa, K.; Morita, T.; Okada, Y.; Ichinose, N.; Hatanaka, M. Chem. Commun. 2010, 46, 6177.

[13] Shi, J.-L.; Yuan, T.; Zheng, M.-F.; Wang, X.-C. ACS Catal. 2021, 11,3040 .

[14] (a) Huang, H.; Yu, C.-G.; Zhang, Y.-T.; Zhang, Y.-Q.; Mariano, P. S.; Wang, W. J. Am. Chem. Soc. 2017, 139, 9799.

(b) Tian, H.-T.; Yang, H.; Tian, C.; An, G.-H.; Li, G.-M. Org. Lett. 2020, 22, 7709 .

(c) Zhao, B.; Shang, R.; Cheng, W.-M.; Fu, Y. Org. Chem. Front. 2018, 5,1782 .

(d) Luo, J.; Zhang, J. ACS Catal. 2016, 6, 873.

(e) Yang, H.; Tian, C.; Qiu, D.-S.; Tian, H.-T.; An, G.-H.; Li, G.-M. Org. Chem. Front. 2019, 6, 2365.

(f) Shang, T.-Y.; Lu, L.-H.; Cao, Z.; Liu, Y.; He, W.-M.; Yu, B. Chem. Commun. 2019, 55, 5408.

(g) Huang, H.; Li, X.-M.; Yu, C.-G.; Zhang, Y.-T.; Mariano, P. S.; Wang, W. Angew. Chem., Int. Ed. 2017, 56, 1500.

(h) Yi, R.-N.; He, W.-M. Chin. J. Org. Chem. 2021, 41, 1267 (in Chinese).

(易荣楠，何卫民，有机化学, 2021, 41, 1267.)

[15] (a) Shirase, S.; Tamaki, S.; Shinohara, K.; Hirosawa, K.; Tsurugi, H.; Satoh, T.; Mashima, K. J. Am. Chem. Soc. 2020, 142, 5668. (b) Bazyar, Z.; Hosseini-Sarvari, M. J. Org. Chem. 2019, 84, 13503 . 